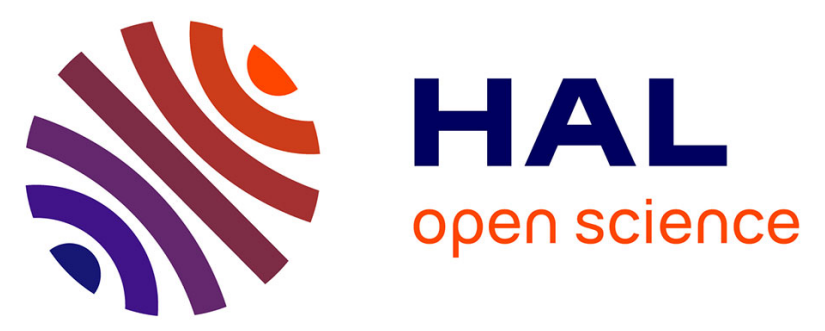

\title{
A Class of Constitutive Relations with Internal Variable Derivatives. Derivation from Homogenization and Initial Value Problem
}

\author{
S. Andrieux, M. Joussemet, E. Lorentz
}

\section{- To cite this version:}

S. Andrieux, M. Joussemet, E. Lorentz. A Class of Constitutive Relations with Internal Variable Derivatives. Derivation from Homogenization and Initial Value Problem. Journal de Physique IV Proceedings, 1996, 06 (C6), pp.C6-463-C6-472. 10.1051/jp4:1996646 . jpa-00254479

HAL Id: jpa-00254479

https://hal.science/jpa-00254479

Submitted on 1 Jan 1996

HAL is a multi-disciplinary open access archive for the deposit and dissemination of scientific research documents, whether they are published or not. The documents may come from teaching and research institutions in France or abroad, or from public or private research centers.
L'archive ouverte pluridisciplinaire HAL, est destinée au dépôt et à la diffusion de documents scientifiques de niveau recherche, publiés ou non, émanant des établissements d'enseignement et de recherche français ou étrangers, des laboratoires publics ou privés. 


\title{
A Class of Constitutive Relations with Internal Variable Derivatives. Derivation from Homogenization and Initial Value Problem
}

\author{
S. Andrieux, M. Joussemet and E. Lorentz
}

Electricité de France, Direction des Etudes et Recherche, Service IMA, Département MMN, Groupe I74, 1 avenue du Général de Gaulle, 92141 Clamart cedex, France

\begin{abstract}
A general framework for deriving and using a class of constitutive laws incorporating spatial gradients of internal variables is presented. It uses two basic ingredients : a derivation of such models by homogenization techniques and a reformulation of the evolution equation at the scale of the whole structure.
\end{abstract}

\section{INTRODUCTION}

When subjected to excessive loads, some materials may exhibit a softening behaviour resulting from the deterioration of their mechanical properties. To model such behaviours, constitutive relations with softening are introduced, for which the size of the domain of reversibility in the stress-space decreases. These models feature a strain localization within the material, in agreement with experiments, but cannot predict the subsequent behaviour because they lead to shear bands the width of which is equal to zero, physically unacceptable and numerically troublesome.

It has been proposed in the literature to overcome these difficulties by adding to the list of internal variable the spatial gradients of some of them. This procedure suffers from lack of firm methodological basis, although some quantitative justifications have been advanced relying on some kind of microscopic analysis.

Therefore, we propose to extend the classical (local) models by introducing the internal state variable first gradients. Given a Jocal model within the context of standard generalized materials, a consistent homogenization procedure is put forward to derive macroscopic free energy and dissipation potentials. The standard generalized character is preserved, with an extended set of state variables, containing not only the strain and the internal variables but also the internal variable derivatives. Nevertheless, when dealing with the whole structure, the independence between the new state variables is lost. We propose then to generalize the constitutive relations, leading to a new variational principle that ensures the Clausius-Duhem inequality at the structure scale.

\section{DERIVATION FROM HOMOGENIZATION}

Let us consider a constitutive model related to clearly identified microscopic mechanisms. We neglect thermal effects and assume the strain to be infinitesimal so that the behaviour is described at the material point level by:

- a set of local internal variables [2], say $(\varepsilon, \alpha)$, where $\varepsilon$ is the strain and $\alpha$ a scalar or tensorial internal variable vector,

- a Helmholtz' free energy function $\Phi \equiv \Phi(\varepsilon, \alpha)$ from which the state equations are derived:

$$
\sigma=\frac{\partial \Phi}{\partial \varepsilon} \quad \mathbf{A}=-\frac{\partial \Phi}{\partial \alpha}
$$

- a dissipation pseudo-potential $\Delta \equiv \Delta(\dot{\alpha})$, to define the internal variable evolution equation within the context of standard generalized materials [1]:

$$
\mathbf{A} \in \partial \Delta(\dot{\alpha}) \text { (os subgradient of } \Delta)
$$


Usually, the derivation of such models is based upon two ingredients: on the one hand, the knowledge and the microscopic representation of the involved dissipative phenomena and, on the other hand, a more or less explicit homogenization process depending on the definition of a local Elementary Representative Volume (ERV). When softening occurs, localization of the internal variables is observed. Because of their high gradients, the usual homogenization strategies may fail since one of their basic assumptions is a small variation of these variables at the microscopic scale. That's why we propose to generalize the homogenization technique in order to obtain some expressions of the macroscopic free energy and dissipation potentials depending on the internal variable first gradients. It leads to consider another ERV, the scale of which is larger than that of the previous one.

\subsection{Homogenization hypothesis}

Each macroscopic point $X$ does hide a substructure in which $\alpha$ may be affected with large variations. Therefore, instead of taking a single cell, say Y, as the ERV, we choose to take into account not only $Y$ but also its neighbouring cells (see Fig. 1). Let us notice that up to now some arbitrariness still remain in the selection of the neighbouring cells. The question of deriving a systematic method of selection is under current works.

The ERV Z, centered on $X$, is then constituted of $N$ cells denoted $Y, i=1, \ldots, N$.

Each cell $Y^{i}$ is attached to:

- the microscopic spacial co-ordinates of the cell centre:

- the free energy:

- the constitutive laws:

- the dissipation pseudo-potential:

$$
\begin{aligned}
& \Phi^{i}={\stackrel{z^{i}}{ }}^{i}\left(\varepsilon^{i}, \alpha^{i}\right) \\
& \sigma^{i}=\frac{\partial \Phi}{\partial \varepsilon}\left(\varepsilon^{i}, \alpha^{i}\right) \quad A^{i}=-\frac{\partial \Phi}{\partial \alpha}\left(\varepsilon^{i}, \alpha^{i}\right) \\
& \Delta^{i}=\Delta\left(\dot{\alpha}^{i}\right)
\end{aligned}
$$

(where fidenotes $\mathrm{f}\left(\mathbf{x}, \mathbf{z}^{\mathrm{i}}\right)$ ).

The relations between the microscopic variables $\left(\varepsilon^{i}, \alpha^{i}\right)$ and the macroscopic ones $(\mathbf{e}(\mathbf{x}), \mathbf{a}(\mathbf{x}) . \nabla \mathbf{a}(\mathbf{x}))$, named localization relations, are chosen in a simple way:

$$
\varepsilon^{i}=\mathbf{e}(\mathbf{x}) \quad \text { and } \quad \alpha^{i}=\mathbf{a}(\mathbf{x})+\nabla \mathbf{a}(\mathbf{x}) \cdot \mathbf{z}^{i}
$$

We denote $[\alpha]$ the vector constituted of the $N$ internal variables of $Z:[\alpha]^{j}=\alpha^{i}$

Let us remind that we aim at describing the macroscopic behaviour through $(\mathbf{e}, \mathbf{a}, \nabla \mathbf{a})$ instead of $([\varepsilon],[\alpha])$. However, Suquet [3] showed that usual homogenization techniques in the context of dissipative materials result in a multiplication of internal variables. Faced with this difficulty, we suggest a method expected to ensure the consistence of the behaviour description by $(\mathbf{e}, \mathbf{a}, \nabla \mathbf{a})$. Such a requirement imposes a "compatibility" condition on the rates of internal variables, that is:

$$
\dot{\alpha}=\dot{\mathbf{a}}(\mathbf{x})+\mathbf{z} \cdot \nabla \dot{\mathbf{a}}(\mathbf{x})
$$

The internal variable gradients are supposed to be small at the scale of the ERV Z:

$$
\nabla \mathbf{a}(\mathrm{x}) \cdot \mathrm{z}^{\mathrm{j}} \ll \mathbf{a}(\mathrm{x}) \quad \forall \mathrm{i}
$$

This approach can be understood as a higher order theory compared with the usual one which neglects the internal variable variations from one cell to its neighbours (periodic or quasiperiodic media).

\subsection{Macroscopic Helmholtz' free energy function: $F \equiv F(e, a, \nabla a)$}

The free energy function $\hat{F}(\mathbf{e},[\alpha])$ at the macroscopic point $X$ is obtained as the average on the ERV $Z$ of the macroscopic free energy function $\Phi$ :

$$
\hat{\mathrm{F}}(\mathbf{e},[\alpha]) \equiv \frac{1}{\mathrm{~N}} \sum_{\mathrm{i}=1}^{\mathrm{N}} \Phi(\mathbf{e}, \alpha)
$$

Given the localization relations (2.1), $\hat{F}$ can be expressed as a function of the macroscopic state variables only: 


$$
\hat{F}(e,[\alpha]) \equiv \frac{1}{N} \sum_{i=1}^{N} \Phi\left(e, a+z^{i}, \nabla \mathbf{a}\right)
$$

Moreover, we assume the function $\Phi$ to be smooth enough to be approximated by a second order Taylor expansion around a with respect to ( $\nabla \mathbf{a} . z)$. It leads to the following expression of the macroscopic free energy function $F(\mathbf{e}, \mathbf{a}, \nabla \mathbf{a})$ (with $n=1,2$ or 3 the dimension of the space):

$$
\begin{aligned}
& \hat{\mathrm{F}}(\mathbf{e},[\alpha]) \stackrel{\text { Taylor }}{\cong} \mathrm{F}(\mathbf{e}, \mathbf{a}, \nabla \mathbf{a})=\Phi(\mathbf{e}, \mathbf{a})+\mathrm{L}_{\mathrm{C}}^{2} \frac{\partial^{2} \Phi}{\partial \mathbf{a}^{2}}(\mathbf{e}, \mathbf{a})\left[\nabla \mathbf{a} . \mathbf{J}^{0} . \nabla \mathbf{a}\right] \\
& \mathbf{J}=\frac{1}{2 N} \sum_{\mathrm{i}=1}^{N}\left(\mathbf{z}^{\left.\mathrm{i} \otimes \mathbf{z}^{i}\right)} \quad \mathrm{L}_{\mathrm{C}}^{2}=\frac{\operatorname{Tr}(\mathbf{J})}{\mathrm{n}} \quad \mathbf{J}^{0}=\frac{\mathbf{J}}{\mathrm{L}_{\mathrm{C}}^{2}}\right.
\end{aligned}
$$

$\mathbf{J}$ is a definite positive second rank tensor with dimension of square of length. It characterizes the size of the ERV through $L_{C}$ and its shape through the adimensional tensor $\mathbf{J}^{0}$.

The expression of the macroscopic free energy function $F(2.4)$ is rather appealing. It is composed of the free energy terms for the homogeneous state and a product of two corrective terms:

- the first one depending only of the local model (second derivative of the local free energy function with respect to the internal variables);

- the second one that takes into account the shape of the ERV (through the tensor $\mathbf{J}$ ) and the loss of homogeneity (through $\nabla \mathbf{a}$ ).

Thus, the model exhibits a dependence on the "texture" of the material, which means the spatial distribution of the microstructure following a pattern defined by the shape of the local ERV Y.

The free energy potential being defined, macroscopic state laws can be expressed as follows, thanks to the standard generalized material property $\left(\mathbf{s}, \mathbf{A}_{2}, \mathbf{A}_{\mathrm{Va}}\right.$ denote respectively the macroscopic stress and the thermodynamic forces associated to $\mathbf{a}$ and $\nabla \mathbf{a})$ :

$$
\mathbf{s}=\frac{\partial \mathrm{F}}{\partial \mathbf{e}} \quad \mathbf{A}_{\mathrm{a}}=-\frac{\partial \mathrm{F}}{\partial \mathbf{a}} \quad \mathbf{A}_{\nabla \mathbf{a}}=-\frac{\partial \mathrm{F}}{\partial \nabla \mathbf{a}}
$$

We can notice that the macroscopic stress is nothing else than the mean stress on the ERV at the firrst order. The same holds for $\mathbf{A}_{\mathbf{a}}$ :

$$
\frac{1}{\mathrm{~N}} \sum_{\mathrm{i}=1}^{N} \sigma^{\mathrm{i}}=\langle\sigma\rangle_{\mathrm{Z}} \cong \mathbf{T} \cong \mathbf{S} \quad \text { and } \quad \frac{1}{\mathrm{~N}} \sum_{\mathrm{i}=1}^{\mathrm{N}} \mathbf{A}^{\mathrm{i}}=\langle\mathbf{A}\rangle_{\mathrm{Z}} \stackrel{\text { Tayior }}{\cong} \mathbf{A}_{\mathbf{a}}
$$

Another remark is of importance. To analyse the dissipation (or equivalently the Clausius-Duhem inequality) at the macroscopic level, i.e. only with the variables $(\mathbf{e}, \mathbf{a}, \nabla \mathbf{a})$, it requires the following equality:

$$
\frac{\partial \hat{F}}{\partial[\alpha]}[\dot{\alpha}] \equiv \frac{\partial F}{\partial \mathbf{a}} \dot{\mathbf{a}}+\frac{\partial F}{\partial \nabla \mathbf{a}} \nabla \dot{\mathbf{a}}
$$

In fact, this equality is satisfied (at the first order) as soon as the localization relation (2.1) and the compatibility condition (2.2) are ensured:

$$
\alpha=\mathbf{a}(\mathbf{x})+\mathbf{z} \cdot \nabla \mathbf{a}(\mathbf{x}) \text { and } \quad \dot{\alpha}=\dot{\mathbf{a}}(\mathbf{x})+\mathbf{z}^{\mathrm{i}} \cdot \nabla \dot{\mathbf{a}}(\mathbf{x})
$$

\subsection{Dissipation potential: $\mathrm{D} \equiv \mathrm{D}(\dot{\mathrm{a}}, \nabla \dot{\mathbf{a}})$}

To derive a macroscopic dissipation potential Da, $\nabla \mathbf{a})$, one can think of a similar approach as above, in which we define the potential $\hat{\mathrm{D}}([\dot{\alpha}])$ as the average of the microscopic one on the ERV Z:

$$
\hat{\mathrm{D}}([\dot{\alpha}])=\frac{1}{N} \sum_{i=1}^{N} \Delta\left(\dot{\alpha}^{i}\right)
$$

However, such a choice does not always preserve the compatibility condition (2.2) and prevents us to describe the state only through the reduced macroscopic variables $(\mathbf{e}, \mathbf{a}, \nabla \mathbf{a})$. We are faced with such a 
difficulty especially when the potential is positively homogeneous of degree one, typical of a material behaviour with threshold.

Precisely, if the state variables verify the localization relation (2.1), a coherent micro/macro model would lead to a rate of $[\alpha]$ to be equivalent to the rate of $(\mathbf{a}, \nabla \mathbf{a})$, when they are derived from the microscopic dissipation potential $\hat{\mathrm{D}}([\dot{\alpha}])$ or the macroscopic one $\mathrm{D}(\dot{\mathbf{a}}, \nabla \dot{\mathbf{a}})$ respectively. That is nothing else than expressing the compatibility condition (2.2).

$$
\begin{array}{c|ccc}
\hat{\mathrm{F}}(\mathbf{e},[\alpha]) ; \quad \hat{\mathrm{D}}^{\mathrm{R}}([\alpha]) & \mathrm{F}(\mathbf{e}, \mathbf{a}, \nabla \mathbf{a}) & : & \mathrm{D}^{\mathrm{R}}(\mathbf{a}, \nabla \mathbf{a}) \\
\mathbf{A}_{\alpha}=\left[\mathbf{A}_{\alpha}^{i}\right]=\frac{\partial \hat{\mathrm{F}}}{\partial[\alpha]} & \mathbf{A}_{\mathbf{a}}=-\frac{\partial \mathrm{F}}{\partial \mathbf{a}} & \mathbf{A}_{\nabla_{\mathbf{a}}}=-\frac{\partial \mathrm{F}}{\partial \nabla \mathbf{a}} \\
\mathbf{A}_{\alpha} \in \partial \hat{\mathrm{D}}^{\mathrm{R}}([\alpha]) & \Leftrightarrow & \left(\mathbf{A}_{\mathbf{a}}, \mathbf{A}_{\nabla_{\mathrm{a}}}\right) \in \partial \mathrm{D}^{\mathrm{R}}(\dot{\mathbf{a}}, \nabla \mathbf{a})
\end{array}
$$

Therefore, we choose to define a regularized dissipation potential on the ERV through a quadratic average, instead of $(2.8)[4]$ :

$$
\hat{D}^{R}([\alpha])=\left\{\frac{1}{N} \sum_{i=1}^{N}\left[\Delta\left(\dot{\alpha}^{i}\right)\right]^{2}\right\}^{1 / 2}
$$

Let us point out that this regularization preserves the positive homogeneity of the microscopic potential if needed.

As previously for the free energy potential, the second step consists in a Taylor expansion truncated at the second order (as soon as $\Delta$ is $C^{2}$ ):

$$
\begin{aligned}
& \hat{\mathrm{D}}^{\mathrm{R}}([\dot{\alpha}]) \equiv \mathrm{D}^{\mathrm{R}}(\dot{\mathbf{a}}, \nabla \dot{\mathbf{a}}) \\
& \mathrm{D}^{\mathrm{R}}(\mathbf{\mathbf { a }}, \nabla \mathbf{\mathbf { a }})=\Delta(\dot{\mathbf{a}})+\mathrm{L}_{\mathrm{C}}^{2}\left[\frac{\partial^{2} \Delta}{\partial \dot{\mathbf{a}}^{2}}+\frac{1}{\Delta(\dot{\mathbf{a}})}\left(\frac{\partial \Delta}{\partial \dot{\mathbf{a}}}\right)^{2}\right]\left[\nabla \dot{\mathbf{a}} \cdot \mathbf{J}^{0} \cdot \nabla \dot{\mathbf{a}}\right]
\end{aligned}
$$

The evolution equations are then (first order accurate):

$$
\mathbf{A}_{\mathbf{a}}=\frac{\partial \Delta}{\partial \dot{\mathbf{a}}} \quad, \quad \mathbf{A}_{\nabla_{\mathbf{a}}}=L_{L}^{2}\left[\frac{\partial^{2} \Delta}{\partial \dot{\mathbf{a}}^{2}}+\frac{1}{\Delta(\dot{\mathbf{a}})}\left(\frac{\partial \Delta}{\partial \dot{\mathbf{a}}}\right)^{2}\right]: \nabla \dot{\mathbf{a}} \cdot \mathbf{J}^{\mathrm{j}}
$$

We can show that (2.9), or identically the compatibility condition (2.2), are verified. Besides, the Clausius-Duhem inequality is ensured, thanks to (2.7), under the usual assumption of convexity of the microscopic dissipation potential.

$$
\begin{aligned}
& \text { s. } \dot{\varepsilon}-\dot{\hat{F}} \cong \frac{\partial F}{\partial \mathbf{a}} \dot{\mathbf{a}}+\frac{\partial F}{\partial \nabla \mathbf{a}} \nabla \dot{\mathbf{a}} \\
& \text { s. } \dot{\varepsilon}-\dot{\hat{F}} \cong \frac{\partial \Delta}{\partial \dot{\mathbf{a}}} \dot{\mathbf{a}}+L_{C}^{2} \quad\left[\frac{\partial^{2} \Delta}{\partial \dot{a}^{2}}+\frac{1}{\Delta(\dot{a})}\left(\frac{\partial \Delta}{\partial \dot{\mathbf{a}}}\right)^{2}\right]\left[\nabla \dot{\mathbf{a}} \cdot \mathbf{J}^{\hat{0}} \cdot \nabla \dot{\mathbf{a}}\right] \geq 0
\end{aligned}
$$

The expression (2.11) is obviously incorrect for a material with a threshold since the potential is not smooth enough. Nevertheless, one is commonly taced with potentials that can be expressed as a smooth function $\delta$ of a norm (denoted $|$.$| ) of the internal variable rates. The metric tensor on the space of the$ internal variables $\alpha$ is denoted by $\mathrm{g}_{\text {.. }}$. When $\delta$ is homogeneous of degree $\mathrm{k}$ :

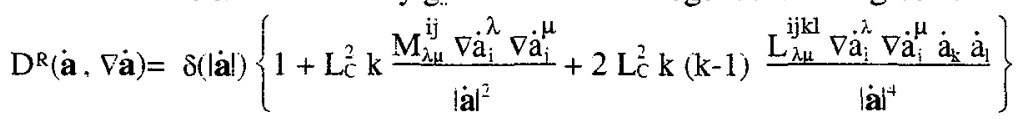

where $\mathbf{M}$ and $\mathbf{L}$ are the tensors which involves only the tensors $\mathbf{J}^{\circ}$ and $\mathbf{g}$ defined by :

$$
\begin{aligned}
& \mathrm{M}_{\lambda \mu}^{\mathrm{ij}}=\mathrm{J}_{\lambda \mu}^{\mathrm{o}} \mathrm{g}_{\mathrm{ij}}
\end{aligned}
$$

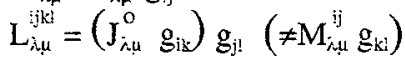




\subsection{Example: Bidimensional elastic crack medium}

We define the microscopic scalar internal variable as the crack density $d=\frac{2 \pi \mathrm{a}^{2}}{\mathrm{~L} \cdot \ell}\left(\mathrm{d} \in\left[0 ; \frac{2 \pi \ell}{\mathrm{L}}\right]\right.$ ) (see figure 1$)$. When it is small $(\mathrm{d} \ll 1)$, we can use the infinite medium assumption. An analytical expression of the compliance tensor $\mathbf{S}(\mathrm{d})$ is then given by Muskhelishvili [5]:

$$
S_{111}=\frac{1}{E_{0 j}} ; S_{2122}=\frac{1+d}{E_{0}} ; S_{1122}=S_{2211}=\frac{-v_{0}}{E_{j}} ; S_{1212}=\frac{2\left(1+v_{0}\right)}{E_{0}}\left(1+\frac{d}{4\left(1+v_{0}\right)}\right)
$$

The other terms are equal to zero. Let us denote the corresponding stiffness tensor by $\mathbf{E}$ (d).

As the crack density is small ( $\mathrm{d}<\frac{2 \pi \ell}{\mathrm{L}}$ ), we truncate $\mathbf{E}(\mathrm{d})$ to the second order. The macroscopic fiee energy is given then by:

$$
\begin{aligned}
& \mathrm{F}(\mathbf{e}, \mathrm{d}, \nabla \mathrm{d})=\frac{1}{2} \mathbf{e} \cdot \mathbf{E}(\mathrm{d}) \cdot \mathbf{e}+\frac{\mathrm{L}_{\mathrm{C}}^{2}}{2} \text { e. } \frac{\partial^{2} \mathrm{E}}{\partial \mathrm{d}^{2}}(\mathrm{~d}) \cdot \mathbf{e} \quad\left({ }^{\nabla} \nabla \mathrm{d} \cdot \mathbf{J}^{\mathrm{J}} \cdot \nabla \mathrm{d}\right) \\
& F(e, d, \nabla d)=\frac{1}{2} \text { e.E(d).e }+L_{c}^{2}\left({ }^{t} \nabla d \cdot J^{\hat{j}} \cdot \nabla d\right) \frac{E_{0}}{\left(1-v_{0}^{2}\right)^{3}}\left(v_{0}^{2} e_{11}^{2}+e_{22}^{2}+2 v_{0} e_{11} e_{22}+\frac{\left(1-v_{0}\right)^{3}}{8} e_{12}^{2}\right)
\end{aligned}
$$

For a lattice of parallel cracks and different orientations, the tensor $\mathbf{J}$ is:

$$
\text { If } \theta=\frac{\pi}{2}: \quad \begin{aligned}
& \mathrm{L}_{\mathrm{C}}^{2}=\frac{\ell^{2}+\mathrm{L}^{2}}{6} \\
& \mathbf{J}^{0}=\frac{2}{\ell^{2}+\mathrm{L}^{2}}\left[\begin{array}{cc}
\ell^{2} & 0 \\
0 & \mathrm{~L}^{2}
\end{array}\right]
\end{aligned} \quad \begin{aligned}
\text { If } \theta=\frac{\pi}{4}: \mathrm{L}_{\mathrm{C}}^{2}=\frac{\ell^{2}+2 \mathrm{~L}^{2}}{6} \\
\mathbf{J}^{0}=\frac{2}{\ell^{2}+2 \mathrm{~L}^{2}}\left[\begin{array}{cc}
\ell^{2}+\mathrm{L}^{2} & \mathrm{~L}^{2} \\
\mathrm{~L}^{2} & \mathrm{~L}^{2}
\end{array}\right]
\end{aligned}
$$

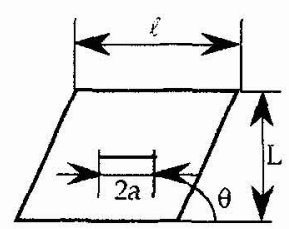

local ERV Y

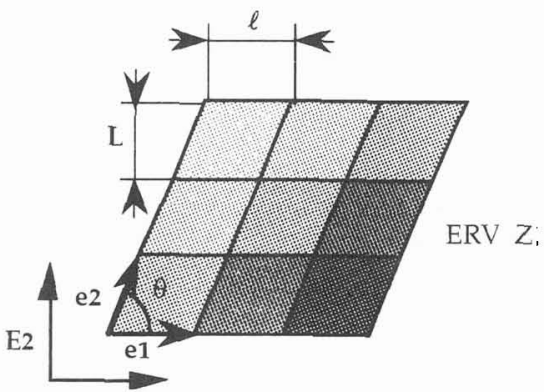

Fig.1 : Characteristic of the lattice of parallel cracks

\subsection{Remarks}

- The expressions for the free energy and the dissipation potential exhibit a dependence on the gradients of the form $\left[{ }^{t} \nabla \mathbf{a} \cdot \mathbf{J}^{6} . \nabla \mathbf{a}\right]$ that ensures frame indifference.

- The standard generalized nature of the 'local' law is preserved by the homogenization procedure.

- If $\nabla \mathrm{a}=0$, the usual expressions of the local constitutive relations are recovered. In particular, if $\mathrm{D}^{\mathrm{R}^{+}}\left(\mathbf{A}_{\mathbf{a}}, \mathbf{A}_{\nabla_{\mathbf{a}}}\right)$ is the Legendre-Fenchel transform of $\mathrm{D}^{\mathrm{R}}(\mathbf{a}, \nabla \mathbf{a})$, we obtain:

$$
\begin{aligned}
& \dot{\mathbf{a}} \in \partial_{\mathbf{A}_{\mathbf{a}}} \mathrm{D}^{\mathrm{R}^{*}}\left(\mathbf{A}_{\mathbf{a}}, \mathbf{A}_{\nabla_{\mathbf{a}}}\right) \text { and } \nabla \dot{\mathbf{a}} \in \partial_{\mathbf{A}_{\nabla_{\mathbf{a}}}} \mathrm{D}^{\mathrm{R}^{*}}\left(\mathbf{A}_{\mathbf{a}}, \mathbf{A}_{\nabla_{\mathbf{a}}}\right) \\
& \text { then: } \quad 0 \in \partial_{\mathbf{A}_{v_{\mathbf{a}}}} D^{R^{*}}\left(\mathbf{A}_{\mathbf{a}}, 0\right)
\end{aligned}
$$


Thus, (2.14) appears as a consistency condition that should be required for any standard generalized model which involves gradient terms. Here, let us note that (2.14) results from the process and is not a priori enforced.

\section{INITIAL VALUE PROBLEM}

Thus far, we have proposed a first order accurate homogenization method that has allowed us to derive free energy and dissipation macroscopic potentials, the arguments of which are the average strain, the internal variables and their first derivatives. This approach is fully consistent at the material point scale within the context of standard generalized materials. Nevertheless, to solve a real initial value problem, the whole structure has to be considered. And there, a paramount difficulty arises: the lack of independence between the internal variable vector and its gradient at the structure scale. Indeed, the state and evolution equations (2.6) and (2.9) define the rate of $\mathbf{a}$ and $\nabla \mathbf{a}$, without taking into account the global (structure scale) constraint that the second one should be the gradient of the first one. In fact, enforcing this global compatibility requirement in addition to the local normality rule (standard generalized materials) do lead to a lack of solution to the constitutive relations. For example, if the internal variable a is a scalar, one can exhibit within the dissipative area of the structure a necessary condition for the existence of a ratea solution which is not a priori verified (where $\mathbf{f}$ is a vector function depending only on the state variables at a material point):

$$
\left(\mathbf{A}_{\mathbf{a}}, \mathbf{A}_{\nabla_{\mathbf{a}}}\right) \in \partial D^{R}(\dot{\mathbf{a}}, \nabla \dot{\mathbf{a}}) \Rightarrow \frac{\nabla \dot{\mathbf{a}}}{\dot{\mathbf{a}}}=\mathbf{f}(\mathbf{e}, \mathbf{a}, \nabla \mathbf{a}) \Rightarrow \nabla \ln (\dot{\mathbf{a}})=\mathbf{f}(\mathbf{e}, \mathbf{a}, \nabla \mathbf{a}) \Rightarrow \operatorname{Rot}(\mathbf{f}(\mathbf{e}, \mathbf{a}, \nabla \mathbf{a}))=0
$$

\subsection{Generalized constitutive relations}

The normality rule appears to be a too restricting condition. Therefore, we propose to weaken it while preserving the standard generalized material formalism at the structure scale. In that way, we introduce two global thermodynamical potential functionals. They are no more functions of pinpoint state variables but depend on the state variable fields, expressed on the whole domain $\Omega$ :

$$
\begin{array}{ll}
\text { Helmholtz free energy } & \mathcal{F}(e, a)=\int_{\Omega} \mathrm{F}(e(\mathbf{x}), a(\mathbf{x}) \cdot \nabla a(\mathbf{x})) \mathrm{d} \mathbf{x} \\
\text { dissipation potential } & \mathcal{D}(\dot{a})=\int_{\Omega} \mathrm{D}(\dot{a}(\mathbf{x}), \nabla \dot{a}(\mathbf{x})) \mathrm{d} \mathbf{x}
\end{array}
$$

As for standard generalized materials, constitutive relations are derived, where the state variables are fields and the associated thermodynamical forces are linear forms on the state fields.

$$
\begin{aligned}
& \text { state equations: } \quad s=\frac{\partial F}{\partial e} \quad A=-\frac{\partial F}{\partial a} \\
& \text { evolution equations: } \quad A \in \partial_{\dot{a}} \mathcal{D}
\end{aligned}
$$

In these generalized constitutive relations, the thermodynamical forces are still meaningful; they are linked to the local thermodynamical forces through the following relations, where $\langle. \mid$.$\rangle denotes the$ duality product:

$$
\begin{array}{ll}
\langle s \mid e\rangle=\int_{\Omega} s(\mathbf{x}) e(\mathbf{x}) \mathrm{d} \mathbf{x} & \forall e \\
\langle A \mid a\rangle=\int_{\Omega}\left[\mathcal{A}_{a}(\mathbf{x}) a(\mathbf{x})+A_{\nabla_{a}}(\mathbf{x}) \nabla a(\mathbf{x})\right] \mathrm{dx} & \forall a
\end{array}
$$

This latter relation enables us to rewrite the evolution equation as a minimization problem for the rate problem: given $\mathcal{A}_{a}$ and $\mathcal{A}_{\mathcal{F}_{a}}$, find a solution of

$$
\operatorname{Inf}_{\dot{a}} \int_{\sqrt{2}}\left[\mathrm{D}(\dot{a}(\mathbf{x}), \nabla \dot{a}(\mathbf{x}))-\mathcal{A}_{a}(\mathbf{x}) \dot{a}(\mathbf{x})-\mathcal{A}_{\nabla a}(\mathbf{x}) \nabla \dot{a}(\mathbf{x})\right] d \mathbf{x}
$$


On a theoretical ground, the existence of solutions to the previous program is proved by Attouch [6], under an assumption of coercivity on $D$, which turns out to be satisfied for positive homogeneous $D$ for index strictly greater than one. In the case of a behaviour with threshold, the problem remains open, even though some convex analysis results may be sufficient to conclude for particular expressions of D.

It should be pointed out that these generalized constitutive relations are equivalent to the local ones if the potentials do not depend on the state variable gradients. Indeed, one can prove (cf. [7]):

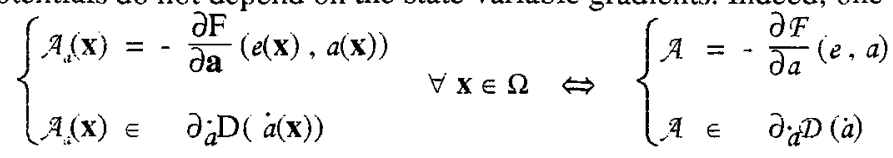

Using (3.1) to (3.5) and the fact that $\mathcal{D}$ is minimal in zero, it can be showed that an extended ClausiusDuhem inequality is ensured, in agreement with the second principle of thermodynamics applied to the whole structure:

$$
\int_{\Omega}[s(\mathbf{x}) \dot{e}(\mathbf{x})-\dot{\mathrm{F}}(e(\mathbf{x}), a(\mathbf{x}), \nabla a(\mathbf{x}))] \mathrm{dx}=\langle s \mid e\rangle-\dot{F}(e, a)=\langle A \mid \dot{a}\rangle \geq 0
$$

Finally, the process which has been followed is nothing else than the "standard generalized" approach extended to the whole structure. Besides, one can notice that if a generalized standard solution (2.6) and (2.9) exists, then it is also solution of the global problem, (3.3) and (3.4).

\subsection{Applications}

Let us consider a classical quasi brittle damage model with a threshold, the yield value of which depends on the damage. Using the results of part 2, a non local model can be deduced. It is applied to an infinite 1D rod, an area of which is weakened, that means its undamaged yield value $\left(k=\eta k_{0}\right)$ is smaller in a ratio $\eta$ than the undamaged yield value elsewhere $\left(\mathrm{k}=\mathrm{k}_{\mathrm{i}}\right)$. Our purpose is to study the rate problem (3.6) which is rather instructive since it exhibits some of the main features of the generalized model.

The microscopic constitutive relations are derived from the free energy and dissipation potentials, where $\mathrm{E}, \mathrm{k}, \gamma, \varepsilon$ and $\alpha$ denote respectively the stiffness, the undamaged yield value (which is not uniform), a hardening parameter, the strain and the damage:

$$
\Phi(\varepsilon, \alpha)=\frac{1}{2} \mathrm{E}(1-\alpha) \varepsilon^{2}+\frac{1}{2} \mathrm{k} \gamma \alpha^{2} \quad \Delta(\dot{\alpha})=\mathrm{k} \mid \dot{\alpha} \mathrm{l}
$$

Elementary calculations show that the constitutive relations are:

- State equations

$$
\sigma=\mathrm{E}(\mathrm{I}-\alpha) \varepsilon \quad \mathrm{G}=\frac{1}{2} \mathrm{E} \varepsilon^{2} \cdot \mathrm{k} \gamma \alpha
$$

- Threshold defined by :

$$
\mathrm{f}(\mathrm{G})=\mathrm{G}-\mathrm{k} \leq 0
$$

- Evolution equations :

$$
\left\{\begin{array}{l}
\dot{\alpha}=0 \text { if } \mathrm{t}<0 \text { or } \dot{\mathrm{t}}<0 \\
\dot{\alpha} \geq 0 \text { if } \mathrm{t}=0 \text { and } \dot{\mathrm{t}}=0
\end{array}\right.
$$

Following part 2, the macroscopic potentials are (2.4) and (2.14), where $L_{c}$ denotes the internal length defined in (2.5):

$$
\begin{aligned}
& F(e, a, \nabla a)=\frac{1}{2} E(1-a) e^{2}+\frac{1}{2} k \gamma\left(a^{2}+2 L_{i}^{2} \nabla a^{2}\right) \\
& D(\dot{a}, \nabla \dot{a})=k \operatorname{aj}\left(1+L_{c}^{2} \frac{\nabla \dot{a}^{2}}{\dot{a}^{2}}\right)
\end{aligned}
$$


Let us assume that the considered state is undamaged ( $e=e_{i}$ constant, $\left.a=0\right)$. The thermodynamical forces associated with the damage and its gradient are:

$$
A_{a}(x)=\frac{1}{2} \operatorname{Ee}_{i}^{2} \quad A_{\nabla_{2}}(x)=0 \quad \forall x \in \Omega
$$

For this state, the rate problem (3.6) can be written, thanks to the symmetry:

$$
\operatorname{Inf} \int_{\dot{a}}^{+\infty}\left[k(x) \mid a d\left(1+L_{c}^{2} \frac{\nabla \dot{a}^{2}}{a^{2}}\right)-A_{a} a\right] d x
$$

First, we can notice that as $A_{a}$ is positive, the rate of damage will be positive without needing to enforce it. Besides, since D is positive homogeneous of degree one, the rate of damage will only be defined modulo a positive multiplicative constant $\lambda$. The effective minimization (which is not detailed here) leads to a yield value $k_{i} \eta^{y}$ (where $\eta^{y}$ is obtained through the transcendental equation 3.11) that depends on the material and the geometry. While $A_{a}<k_{0} \eta^{y}$, damage does not occur.

$$
\tan \left(\frac{\ell_{e}}{2 L_{c}} \sqrt{\frac{\eta^{y}-\eta}{\eta}}\right)=\sqrt{\frac{1-\eta^{y}}{\eta\left(\eta^{y}-\eta\right)}}
$$

On the other hand, when $A_{a}$ reaches $k_{j} \eta y$, damage appears with a rate given by (3.12) and presented on figure 2 .

$$
\left\{\begin{array}{ll}
\dot{a}(\mathrm{x})=\lambda\left(1+\cos \frac{\mathrm{x}}{\mathrm{L}_{\mathrm{c}}} \sqrt{\frac{\eta^{y}-\eta}{\eta}}\right) & \text { if } \mathrm{x}<\ell_{\mathrm{e}} \\
\dot{a}(\mathrm{x})=\lambda\left(1+\cos \frac{\ell_{e}}{\mathrm{~L}_{c}} \sqrt{\frac{\eta^{y}-\eta}{\eta}}\right) \exp \left(-\frac{\mathrm{x}-\ell_{e}}{\mathrm{~L}_{\mathrm{c}}} \sqrt{1-\eta^{y}}\right) & \text { if } \mathrm{x}>\ell_{\mathrm{e}}
\end{array} \quad \lambda \geq 0\right.
$$

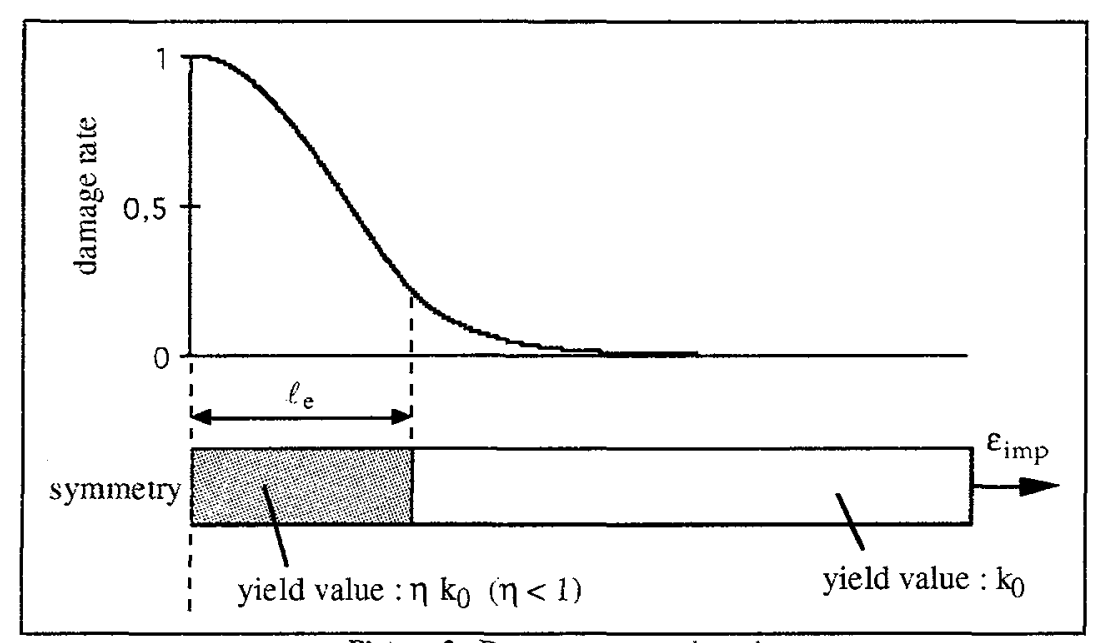

Picture 2 - Damage rate over the rod

The solution is worth being examined for two borderline cases:

- On the one hand, when the length of the weakened area is small compared to the material characteristic length $\left(l_{\mathrm{e}}<<\mathrm{L}_{c}\right)$, the structure tends to respond as though there were no weakened area at all. Damage occurs homogeneously as soon as the yield value $\mathrm{k}_{j}$ is reached:

$$
\eta^{y}=1 \quad \dot{a}(\mathrm{x})=\lambda \quad \lambda \in \mathbb{R}^{+}
$$


- On the other hand, when the material characteristic length is small compared to the size of the weakened area $\left(\mathrm{L}_{\mathrm{c}}<<\ell_{\mathrm{e}}\right)$, damage tends to occur only in the weakened area as soon as the yield value $\eta \mathrm{k}_{\mathrm{i}}$ is reached:

$$
\eta^{y}=\eta \quad\left\{\begin{array}{ll}
\dot{a}(\mathrm{x})=\lambda\left(1+\cos \frac{\pi \mathrm{x}}{\ell_{e}}\right) & \text { si } \mathrm{x}<\ell^{\prime} \\
\dot{a}(\mathrm{x})=0 & \text { si } x>\ell_{e}
\end{array} \quad \lambda \in \mathbb{R}^{+}\right.
$$

Some remarks apply to the rate problem:

- it can be noticed that the yield value depends not only on $\mathrm{k}_{\mathrm{i}}$ but also on $\eta$ and $\ell_{\mathrm{e}}$. Generally speaking, it can be said that the threshold has become global, depending on the whole structure. Besides, it should be pointed out that $\lambda$, commonly named plastic multiplier, is no more a field but a single positive scalar for the whole structure. Thus, the difference between formulation (3.6) and the variational principle proposed by Mühlhaus [8] appears clearly: it is not a straightforward variational formulation of the material point constitutive relations.

- When the yield value is reached, the whole rod does damage, and not only a finite part which would include the weakened area. It may be explained by the Neumann natural boundary conditions that are not a priori stated but are derived from the minimization (3.10). The question of the boundary conditions imposed to the damage field (Dirichlet or Neumann), an issue often met (even tacitly) in the literature, is here answered.

- The rate problem does not exhibit any bifurcation. To detect them (if there are any), one should analyse the incremental problem which takes into account the variations of the reversibility domain.

\subsection{Incremental variational principle}

To deal with a real structure computation involving such generalized constitutive relations, two kinds of equations have to be solved simultaneously: on the one hand, those describing the behaviour (3.3) and (3.4), and, on the other hand, the equilibrium equation (where $\mathrm{W}_{\mathrm{exi}}$ denotes the external virtual work and $\nu_{\mathrm{KA}}$ the kinematically admissible displacement rates):

$$
\langle s \mid e(v)\rangle=\mathrm{W}_{\mathrm{ext}}(v) \quad \forall v \in \mathcal{V}_{\mathrm{KA}}
$$

To solve numerically these equations, an implicit time-step algorithm is chosen. It leads to the following incremental problem:

$$
\begin{aligned}
<s+\Delta s \mid e(v)> & =\left(\mathrm{W}_{\mathrm{ext}}+\Delta \mathrm{W}_{\mathrm{ex}}\right)(v) \\
s+\Delta s & =\frac{\partial F}{\partial e}(e+\Delta e, a+\Delta a) \\
-(A+\Delta A) & =\frac{\partial F}{\partial a}(e+\Delta e, a+\Delta a) \\
\mathcal{A}+\Delta A & =\partial_{a} D\left(\frac{\Delta a}{\Delta \mathrm{l}}\right)
\end{aligned}
$$

As the potentials are convex, this system can be equivalently expressed with a variational principle, cf. [6]. If $u$ denotes the displacement, we define a potential, named total energy $\mathcal{E}$ :

$\mathcal{E}(\Delta u, \Delta a)=F(e+\Delta e, a+\Delta a)+\Delta \mathrm{t} D\left(\frac{\Delta a}{\Delta \mathrm{t}}\right)-\left(\mathrm{W}_{\mathrm{ext}}+\Delta \mathrm{W}_{\mathrm{ext}}\right)(\Delta u)$

Then, the displacement and internal variable increments, denoted by $\Delta u$ and $\Delta a$, are given by the two minimization programs:

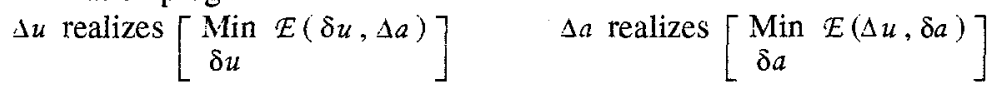

- If $\mathcal{E}$ is not convex with respect to the couple $(\Delta u, \Delta a)$, then a solution of (3.15) does not necessary realizes a minimum of $\mathcal{E}$. 
- In this case, existence and uniqueness of solutions are not ensured. Bifurcations and ultimate loads are possible, in agreement with the experiments on materials that exhibit a softening behaviour.

- This variational principle is close to the energetic approach presented in [9]. In the same way, a stability condition of the incremental solution can be expressed: a couple $(\Delta u, \Delta a)$ is said to be stable if it realizes a minimum of the total energy functional $\mathcal{E}$.

\section{CLOSURE}

Finally, this approach is a strategy among others to deal with localization phenomena. However, it answers some questions which are often raised in the related literature.

- The homogenization technique allows a systematic derivation of constitutive relations which include internal variable first gradients. The obtained potentials are the sum of the classical (local) potentials and a corrective term, quadratic in the internal variable derivatives. This latter does not introduce a scalar (so called characteristic) length but a full symmetric second order tensor, characteristic of the shape of the cells.

- The spirit of generalized standard materials is preserved along the derivation, so that the ClausiusDuhem inequality is naturally ensured.

- No specific (arbitrary) boundary conditions are imposed to the damage field. They are obtained through the minimization program.

- As pointed out in [8], some non local models exhibit difficulties (localization) in the hardening phase, even though they disappear in the softening phase. Thanks to the variational principle (3.15), such issues are avoided here.

At last, some questions remain unanswered among which two at least are of importance: first, the choice of a representative neighbouring cell set in the homogenization technique is not clear. Then, the existence of solution to the rate problem (3.6) for materials with a threshold.

\section{References}

[1] Halphen B., Nguyen Q. S., "Sur les matériaux standards généralisés", J. de mécanique, 14 (1975), pp. 39-63.

[2] Coleman B. D., Gurtin M. E., "Thermodynamics with internal state variables", J. of Chemical Phys., 47 (2) (1967), pp. 597-613.

[3] Suquet P., "Plasticité et homogénéisation", Thèse d'état, Université Paris 6, (1982).

[4] Michel J.-C., "Homogénéisation de matériaux élastoplastiques avec cavités", Thèse, Université Paris 6 (1984).

[5] Bui H. D., "Mécanique de la rupture fragile", (Masson, 1978).

[6] Attouch H., Damlamian A., "Application des méthodes de convexité et monotonie à l'etude de certaines équations quasi-linéaires", in Proc. Roy. Soc. Edinburgh, 79 A (1977), pp 107-129.

[7] Ekeland I., Temam R., "Analyse convexe et problèmes variationnels", Etudes mathématiques (Dunod, Gauthier-Villars, Bordas, 1974).

[8] Mühlhaus H., Aifantis E., "A variational principle for gradient plasticity", Int. J. Solids Struct., 28 (7) (1991), pp. 845-857.

19] Francfort G., Marigo J.-J., "Stable damage evolution in a brittle continuous medium", Eur. J. Mech. A/Solids, 12 (2) (1993), pp. 149-189. 